
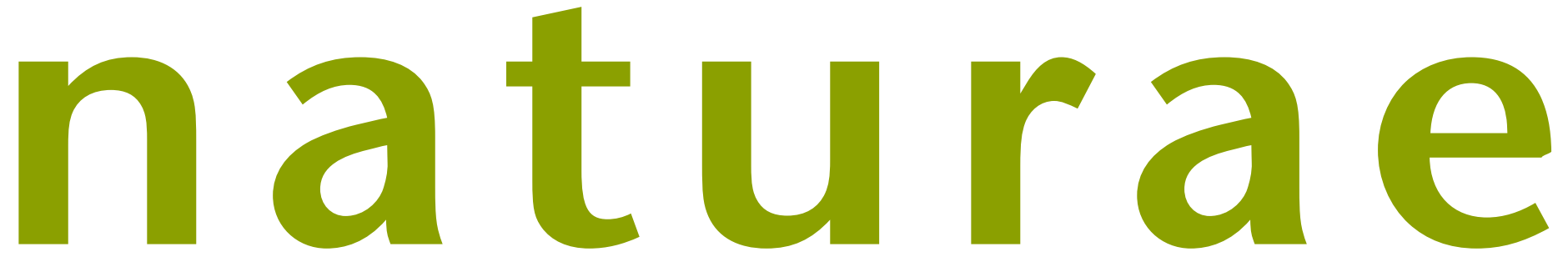

$2020 \cdot 16$

COLLOQUE NATIONAL DE MALACOLOGIE CONTINENTALE, comme indicateur des changements globaux

Damien COMBRISSON, Amélie SAILLARD \& Wilfried THUILLER 
Directeur de LA Publication / Publication diRECTOR: Bruno David,

Président du Muséum national d'Histoire naturelle

RÉDACTEUR EN CHEF / EDITOR-IN-CHIEF: Jean-Philippe Siblet

ASSISTANTE DE RÉDACTION / ASSISTANT EDITOR: Sarah Figuet (naturae@mnhn.fr)

Mise EN PAGE / PAGE LAYOUT: Sarah Figuet

COMITÉ SCIENTIFIQUE / SCIENTIFIC BOARD:

Luc Abbadie (UPMC, Paris)

Luc Barbier (Parc naturel régional des caps et marais d'Opale, Colembert)

Aurélien Besnard (CEFE, Montpellier)

Vincent Boullet (Expert indépendant flore/végétation, Frugières-le-Pin)

Hervé Brustel (École d'ingénieurs de Purpan, Toulouse)

Patrick De Wever (MNHN, Paris)

Thierry Dutoit (UMR CNRS IMBE, Avignon)

Éric Feunteun (MNHN, Dinard)

Romain Garrouste (MNHN, Paris)

Grégoire Gautier (DRAAF Occitanie, Toulouse)

Olivier Gilg (Réserves naturelles de France, Dijon)

Frédéric Gosselin (Irstea, Nogent-sur-Vernisson)

Patrick Haffner (UMS PatriNat, Paris)

Frédéric Hendoux (MNHN, Paris)

Xavier Houard (OPIE, Guyancourt)

Isabelle Leviol (MNHN, Concarneau)

Francis Meunier (Conservatoire d'espaces naturels - Hauts-de-France, Amiens)

Serge Muller (MNHN, Paris)

Francis Olivereau (DREAL Centre, Orléans)

Laurent Poncet (UMS PatriNat, Paris)

Nicolas Poulet (OFB, Vincennes)

Jean-Philippe Siblet (UMS PatriNat, Paris)

Laurent Tillon (ONF, Paris)

Julien Touroult (UMS PatriNat, Paris)

COUVERTURE / COVER:

La Meije et le Râteau se reflètent dans le lac noir sur le plateau d’Emparis. Crédit photo: Parc national des Écrins - P. Saulay.

\section{MUSÉuM Nantes
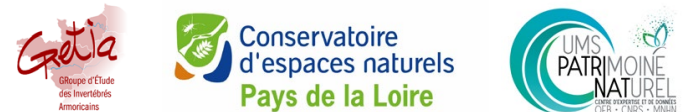

Les Publications scientifiques du Muséum publient aussi / The Museum Science Press also publish:

Adansonia, Zoosystema, Anthropozoologica, European Journal of Taxonomy, Geodiversitas, Cryptogamie sous-sections Algologie, Bryologie, Mycologie, Comptes Rendus Palevol.

Diffusion - Publications scientifiques Muséum national d'Histoire naturelle

CP 41 - 57 rue Cuvier F-75231 Paris cedex 05 (France)

Tél. : 33 (0)1 40794805 / Fax: 33 (0)1 40793840

diff.pub@mnhn.fr / http://sciencepress.mnhn.fr

(C) Publications scientifiques du Muséum national d'Histoire naturelle, Paris, 2020

ISSN (électronique / electronic): 1638-9387 


\title{
La prise en compte de la malacofaune continentale comme indicateur des changements globaux
}

\author{
Damien COMBRISSON \\ Parc national des Écrins, Domaine de Charance, F-05000 Gap (France) \\ damien.combrisson@ecrins-parcnational.fr \\ Amélie SAILLARD \\ Wilfried THUILLER \\ Laboratoire d'Écologie alpine, Université Grenoble Alpes, Université Savoie Mont Blanc, CNRS, \\ Université Grenoble Alpes, cs 40700, F-38058 Grenoble cedex (France) \\ amelie.saillard@univ-grenoble-alpes.fr \\ wilfried.thuiller@univ-grenoble-alpes.fr
}

Soumis le 4 juillet 2019 | Accepté le 9 avril 2020 | Publié le 18 décembre 2020

MOTS CLÉS

Bio-indicateur, changement climatique,

Mollusques

continentaux

Parc national des Écrins,
Zone Atelier Alpes,

ORCHAMP,

marge glaciaire.
Combrisson D., Saillard A. \& Thuiller W. 2020. - La prise en compte de la malacofaune continentale comme indicateur des changements globaux, in Léonard L. (éd.), Colloque national de malacologie continentale, Nantes, 6 et 7 décembre 2018. Naturae 2020 (16): 225-230. https://doi.org/10.5852/naturae2020a16

\section{RÉSUMÉ}

La compréhension et la prévision des conséquences des changements environnementaux mondiaux (changement climatique et d'affectation des terres, dépôts atmosphériques, invasions d'espèces exotiques) sur la biodiversité et le fonctionnement des écosystèmes figurent parmi les principaux thèmes de recherche scientifique. Le groupe régional d'experts sur le climat en région Sud Provence-Alpes-Côte d'Azur (GREC-SUD) a rapporté en 2018, une élévation des températures observées actuellement dans les Alpes deux fois plus importante que celle notée en plaine. Le suivi de ces processus est à privilégier dans les espaces protégés. La pérennité des parcs nationaux ainsi que la présence d'agents qualifiés sur le terrain sont autant d'éléments facilitant la mise en œuvre d'études opérationnelles sur ces territoires d'exception. Au travers de deux exemples développés au sein du Parc national des Écrins, nous présentons deux protocoles distincts ayant pour cadre l'étude des changements globaux et intégrant les Mollusques continentaux en tant qu'indicateur de ces évolutions. Le premier de ces dispositifs concerne la mise en place de transects altitudinaux permettant de caractériser les peuplements malacologiques (diversité spécifique et abondance) intégrés au sein de l'observatoire ORCHAMP. Ce dispositif de recherche multi partenarial de la Zone Atelier Alpes (ZAA) a pour objectif de suivre la dynamique des écosystèmes de montagne face aux changements globaux (climat, pollution, changements de pratiques, etc.). Le second dispositif concerne un protocole d'étude portant sur la dynamique biologique des marges glaciaires, initié dans le cadre du programme européen ALCOTRA «CclimatT » dont l'objectif est d'appréhender les organismes et communautés d'organismes qui colonisent des zones récemment libérées des glaces à l'échelle du Quaternaire. Au travers de ces deux exemples, les Mollusques continentaux sont intégrés dans le cadre de recherches pluridisciplinaires privilégiant la compréhension des dynamiques locales à partir d'une approche multi-taxons. Le cadre de ces études ainsi que les méthodes employées sont exposés afin d'illustrer de façon concrète l'utilisation de la malacofaune en tant que marqueur des changements globaux. 


KEY WORDS
Bio-indicator,
Climate change,
Continental mollusks,
Ecrins National Park,
Zone Atelier Alpes,
ORCHAMP,
Glacier Margin.

KEY WORDS

Bio-indicator Contal mollusks, crins National Park, ORCHAMP, Glacier Margin.

\begin{abstract}
Taking into account the continental malacofauna as indicator of global changes.

Understanding and predicting the consequences of global environmental changes (climate and land use change, atmospheric deposition, alien invasions) on biodiversity and ecosystem functioning are among the main scientific research topics. In the Alps, the rise in temperatures currently observed is twice as great as that observed in the plains. The monitoring of these modifications is to be favored in protected areas. The long-term survival of national parks and the presence of qualified agents on the territory are elements facilitating the implementation of operational studies on these exceptional territories. Through two examples developed within the Ecrins National Park, we present two distinct protocols for the study of global changes and integrating continental mollusks as an indicator of these evolutions. The first of these measures concerns the establishment of altitudinal transects allowing the characterization of malacological populations (specific diversity and abundance) integrated within a multi-partner research system, ORCHAMP, in which physical (climate, soil, land use) and biotic measurements are conducted to better understand, in time and space, the dynamics between climate, land use and biodiversity of the mountain ecosystems. ORCHAMP is coordinated by the Laboratoire d'Écologie Alpine (LECA), as part of the Zone Atelier Alpes, a French eLTER site. The second device concerns a study protocol on the biological dynamics of the ice margins initiated within the framework of the European program ALCOTRA "CclimatT" whose objective is to apprehend the organisms and communities of organisms that colonize areas recently liberated from ice cream at the Quaternary level. Through these two examples, continental mollusks are integrated in the framework of multidisciplinary research privileging the understanding of local dynamics from a multi-taxon approach. The framework of these studies as well as the methods employed are presented in order to concretely illustrate the use of malacofauna as a marker of global changes.
\end{abstract}

\section{INTRODUCTION}

Créé depuis 1973, le Parc national des Écrins (PNE), répartit sur les départements de l'Isère (38) et des Hautes-Alpes (05), couvre une superficie de 271000 hectares en considérant la zone cœur et l'aire d'adhésion. Ce territoire de haute montagne est fortement influencé par la Durance. Cette rivière draine des influences méditerranéennes au sud, tandis que la Romanche, au nord du massif, se caractérise par un climat plus humide et plus froid typique des Alpes du Nord. Suivant cette ligne de partage, les Écrins se situent donc comme un massif de transition, organisant la synthèse d'influences climatiques méditerranéennes et alpines.

Depuis près de 50 ans d'existence, l'acquisition des connaissances taxonomiques au sein du PNE se poursuit et se reporte maintenant sur des groupes d'espèces liés à la «biodiversité ordinaire», qui reste paradoxalement mal connue (ex.: la micro et macrofaune du sol). C'est dans ce contexte que l'inventaire de la malacofaune continentale a été mis en œuvre au sein du parc à partir des années 2014 (Combrisson \& Maillard 2016). Dès lors et jusqu'à la fin de l'année 2018, près de 2700 données d'occurrence se reportant à 157 taxons ont été relevées sur cet espace, essentiellement obtenues par l'observation des agents de terrain. Les besoins de connaissance sur ce groupe restent encore importants, en particulier sur la partie nord du massif et les territoires de haute montagne formés par la zone cœur du parc qui restent actuellement sous-prospectés.

Au-delà des besoins d'inventaires portant sur les Mollusques continentaux, les espèces sténoèces de ce groupe, en particulier les Gastéropodes terrestres, sont également de précieux indicateurs des conditions stationnelles. Ainsi les peuplements malacologiques sont étroitement liés à la structure et à la composition de la végétation (e.g. Magnin et al. 1995).

Les Gastéropodes sont pour la plupart des espèces phytophages et/ou détritivores: en se déplaçant sur le sol (qu'ils contribuent à enrichir en participant aux échanges avec celui-ci), ils intègrent également de multiples sources et voies de contamination (Vaufleury 2015; Louzon et al. 2020). Différentes études conduites sur l'Escargot petit-gris Cornu aspersum (O. F. Müller, 1774) mettent en évidence le lien entre la biodisponibilité des contaminants du milieu (sol, plantes, air) et l'accumulation de ces polluants dans les animaux (Baurand et al. 2014).

Leur faible mobilité et la possibilité de conservation des coquilles en milieu naturel représentent un atout pour la prise en compte des Mollusques dans le cadre de suivis portant sur la dynamique d'évolution des écosystèmes, comme en témoigne les apports récents de la paléomalacologie (Rousseau et al. 1992; Limondin-Lozouet 2011).

\section{PRÉSENTATION DES DISPOSITIFS D'ÉTUDES MIS EN CEUVRE}

\section{LE PROGRAMME ORCHAMP}

Depuis 2016, la Zone Atelier Alpes (ZAA) structure un ensemble de cinq dispositifs d'observations et de recherches pluridisciplinaires, nommés Sentinelles des Alpes. Ces dispositifs portent sur les trajectoires et le fonctionnement des socio-écosystèmes des Alpes dans un contexte de changements climatiques glo- 


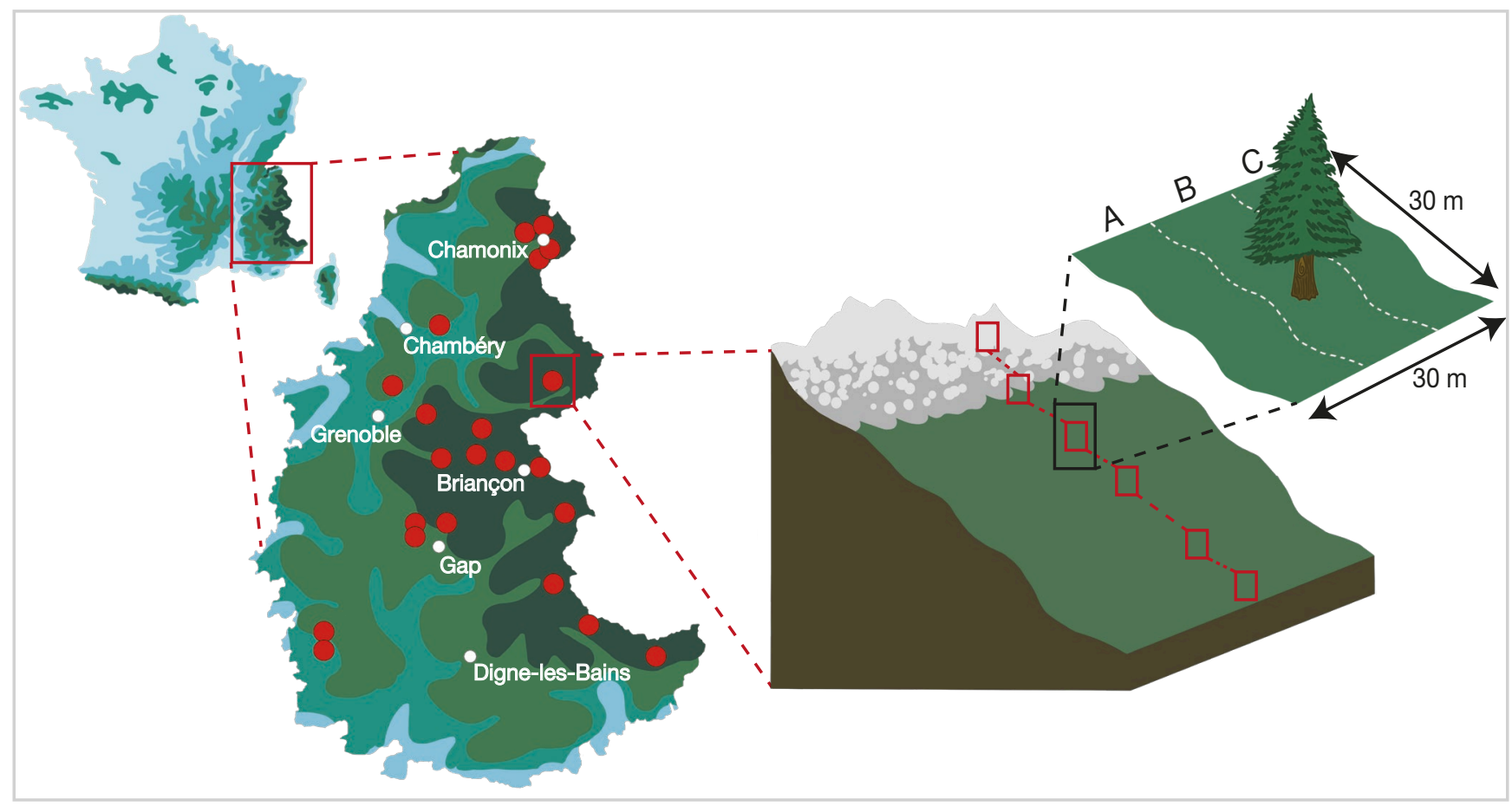

FIG. 1. - Les différents sites du dispositif ORCHAMP de 2016 à 2019.

baux et de mutations socio-économiques des territoires de montagne. L'observatoire spatio-temporel de la biodiversité et du fonctionnement des socio-écosystèmes de montagne, dit ORCHAMP, est l'un de ces dispositifs (https://orchamp.osug. $\mathrm{fr} /$ home, dernière consultation le 28 juillet 2020).

Le dispositifORCHAMP est piloté par le Laboratoire d'Écologie alpine (LECA) de Grenoble. Dans le détail, il s'étend le long de gradients altitudinaux, dont l'amplitude est comprise entre 800 et 1200 mètres de dénivelé, à partir desquels sont répartis des placettes permanentes de $900 \mathrm{~m}^{2}$ espacées tous les 200 mètres d'altitude. Le dispositif est constitué de près de 25 sites en 2019 (Fig. 1).

Au sein de ces placettes, un socle commun de suivis porte sur la végétation (richesse spécifique, abondance des plantes, suivis des forêts et du bois mort), l'étude physico-chimique du sol et des activités s'y déroulant (activités enzymatiques), mais aussi sur la caractérisation de la biodiversité du sol par l'utilisation des méthodes d'identification d'ADN environnemental. Ces mesures sont accompagnées de la pose de capteur de température de sub-surface des sols (type HOBO) pour l'ensemble de la période de suivi. Le suivi temporel de tous ces sites se fait selon une stratégie dite de « rotative panels » où les gradients à rééchantillonner sont tirés au sort chaque année.

En dehors du socle commun de suivis des sites ORCHAMP, des protocoles de suivi supplémentaires peuvent être proposés aux opérateurs locaux, comme le suivi de la malacofaune terrestre des sites.

Le protocole malacologique se base sur une approche quantitative du peuplement à travers la mise en place de six quadrats de $30 \times 30 \mathrm{~cm}$. Ces quadrats sont projetés en amont et en aval de la ligne centrale de la station servant pour le suivi de la végétation. La litière présente dans ces quadrats est récol- tée et pré-tamisée in situ sur un tamis de $5 \mathrm{~mm}$ de vide de maille. La litière tamisée est ensachée et traitée en laboratoire. Ce protocole est complété par une recherche à vue des Mollusques (approche qualitative), effectuée par un observateur dans toute la placette pendant 30 minutes.

\section{LE PROGRAMME «CHANGEMENT CLIMATIQUE DANS LE TERRITOIRE TRANSFRONTALIER» (CCLIMATT)}

Les Mollusques continentaux sont pris en compte dans le programme Interreg "Changement climatique dans le Territoire transfrontalier» (CCLimaTT), entre l'Italie et la France.

Parmi les nombreuses actions mises en œuvre au sein de CCLimaTT, un suivi permet d'appréhender la dynamique de colonisation des marges périglaciaires du Glacier Blanc dans le Parc national des Écrins. Ce suivi privilégie une approche multi-taxons, qui a été mise en œuvre pendant l'été 2019. Cette étude est menée en collaboration avec l'Institut pour la Recherche et le Développement (IRD) et l'Institut national de Recherche pour l'Agriculture, l'Alimentation et l'Environnement (INRAE). Elle se base sur le calage des successions chronologiques connues du retrait du front glaciaire, grâce aux restitutions photogrammétriques réalisées par clichés aériens. Ce travail préparatoire accompli par l'IRSTEA permet de disposer d'une série de séquences chronologiques ou "chrono séquences" au sein desquelles des quadrats de $1 \mathrm{~m}^{2}$ permettant le suivi de la végétation ont été mis en place. De façon concomitante, le suivi des Fourmis, Orthoptères, Coléoptères et des Araignées a été réalisé par des recherches à vue à l'intérieur de chaque quadrat, tandis que les Mollusques continentaux ont été recherchés à vue à l'intérieur d'un cercle de quatre mètres de diamètres centré sur le quadrat de suivi de la végétation. 


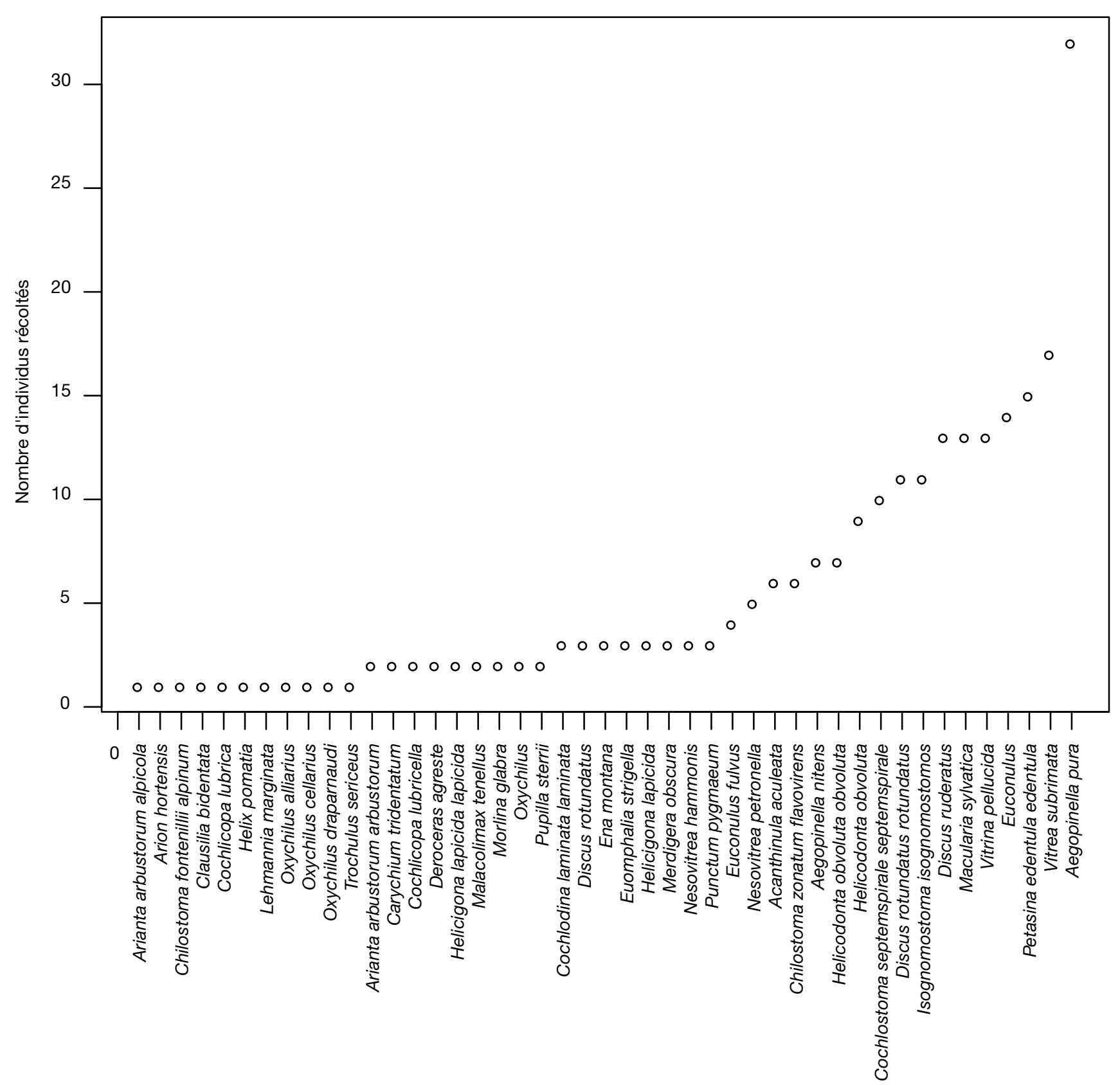

FIG. 2. - Nombre d'individus par espèce sur l'ensemble des gradients d'ORCHAMP.

\section{PREMIERS RÉSULTATS}

Les premiers résultats disponibles ne concernent que ceux de l'observatoire ORCHAMP, l'étude portant sur les marges glaciaires n'est pas encore exploitée.

Intégré au programme ORCHAMP pour la première fois en 2018, le dispositif de suivi de la malacofaune continentale a été mis en œuvre sur quatre des six gradients envisagés cette année-là. Deux suivis ont été réalisés dans le Parc naturel régional des Bauges sur les sites d'Armenaz et de Peclod (73), un troisième suivi a été réalisé sur un versant sud de la chaîne du Dévoluy, à Montmaur (05) ainsi qu’à l'intérieur de la Réserve intégrale (RI) du Lauvitel (38) situé dans le PNE.
Les transects réalisés avec l'approche quantitative et qualitative ont permis de dresser une liste composée de 45 espèces sur la base de 246 individus récoltés. Les espèces les plus abondantes sont: la Petite luisantine Aegopinella pura (Alder, 1830), la Cristalline méridionale Vitrea subrimata (Reinhardt, 1871), la Veloutée alpine Edentiella edentula (Draparnaud, 1805) ou bien encore l'Escargot des forêts Macularia sylvatica (Draparnaud, 1801) (Fig. 2).

Sous l'effet de l'altitude, on observe également une diminution de la diversité spécifique en même temps qu'une diminution du nombre d'individus (Fig. 3). On observe une faible abondance sur le site de Montmaur, tandis que les transects de Pecloz et du Lauvitel apparaissent a priori plus favorables aux Mollusques. 


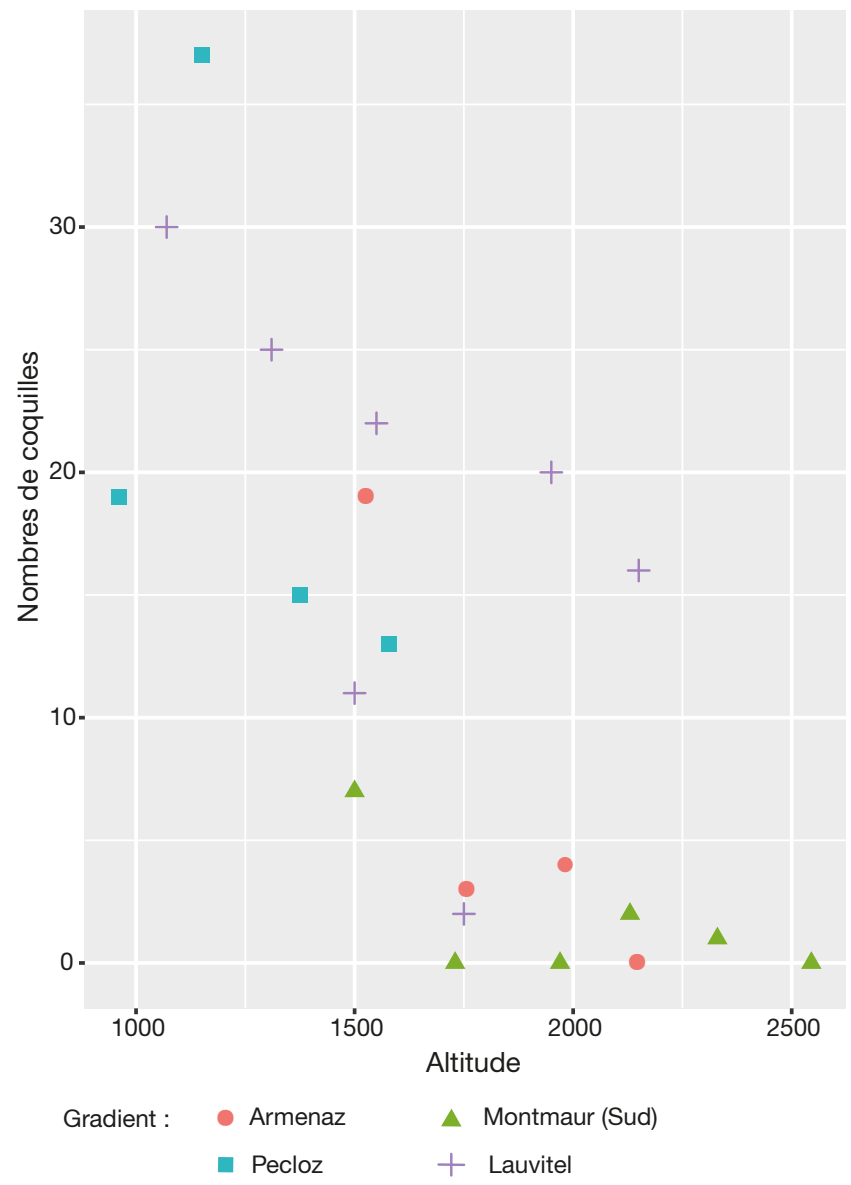

FIG. 3. - Nombre d'individus observés en fonction de l'altitude sur les différents gradients.

\section{DISCUSSION}

Au-delà du simple inventaire permettant de mieux appréhender la richesse spécifique au sein de la malacofaune continentale sur le territoire du Parc national des Écrins, la prise en compte des Mollusques terrestres dans le cadre d'études pluridisciplinaires doit permettre d'apporter un éclairage supplémentaire sur l'organisation des biocénoses confrontées aux changements globaux. Avec une faible capacité de locomotion et un turn-over générationnel relativement court, les Mollusques terrestres apparaissent comme un groupe particulièrement utile permettant d'évaluer la dynamique des écosystèmes. Les premiers retours d'expérience sur la mise en œuvre du protocole de malacologie appliquée au dispositif ORCHAMP sont encourageants et permettent de valider les choix méthodologiques retenus. Le renforcement de ce monitoring par l'ajout de nouveaux gradients qui permettra de conduire une analyse plus fiable des résultats, est nécessaire pour assurer la pérennité de cette étude.

Suivant l'exemple des travaux conduits en paléo-malacologie (Magnin \& Martin 2008; Martin et al. 2017), les espèces peuvent être rassemblées en communautés d'espèces partageant une ou plusieurs exigences écologiques strictes (sténoèce), ainsi leur apparition/disparition donne une lecture sur les trajectoires empruntées par les écosystèmes (fermeture de milieux, etc.). Les activités anthropiques telles que le pastoralisme en montagne devront nécessairement s'adapter aux contraintes liées aux changements globaux et viendront amplifier les modifications sur la structure des habitats (ouverture des milieux, enrichissement des sols, tassement des sols, etc.) et des biocénoses associées.

La diminution de la richesse spécifique au fur et à mesure de l'augmentation de l'altitude témoigne de contraintes environnementales climatiques particulièrement exigeantes (durée d'enneigement, exposition UV, amplitude thermique journalière, etc.). Les Mollusques présents dans les zones de hautes altitudes comprises entre 2000 et 3000 mètres pourraient subir de plein fouet les conséquences de l'élévation des températures (Pearce \& Megan 2013), en particulier les espèces alpines tel que l'Hélicon des Préalpes Delphinatia fontenillii alpina (Michaud, 1831) ou la Semilimace recouverte Eucobresia glacialis (Forbes, 1837).

Dans les habitats forestiers, la densité et la richesse spécifique des Mollusques continentaux sont directement influencées par la structure des habitats; en particulier la couverture herbacée, le diamètre moyen des arbres, l'âge du peuplement, le volume de bois mort et le volume de bois décomposé (Müller et al. 2005). La résilience des forêts aux changements climatiques est directement liée à la diversité des espèces, au pool génétique des populations ainsi qu'à la taille et la fragmentation des peuplements (Thompson et al. 2009). Dans les Alpes où le couvert forestier est contraint par l'exposition (adret $v s$ ubac) et l'altitude, on observe régulièrement des ensembles mono-spécifiques tels que la pinède à l'adret et la pessière à l'ubac ou bien encore le Mélézin qui domine largement toute la bordure méridionale du Parc national des Écrins. Dans ces conditions, quelle sera la résilience de ces écosystèmes face aux changements directs (anthropiques) ou indirects (climatiques)?

Ce premier état des lieux permettra d'apprécier les dynamiques en œuvre au sein des écosystèmes de montagne particulièrement affectés par l'ampleur des changements climatiques.

\section{Remerciements}

Nos remerciements s'adressent aux membres de l'observatoire ORCHAMP et par anticipation pour les suivis qui seront déployés dans les années à venir. À l'équipe du LECA de Wilfried Thuiller (directeur de recherche au Laboratoire d'écologie alpine en charge du programme ORCHAMP), Amélie Saillard et Julien Renaud. À Fabien Anthelme (directeur de recherche à l'IRD), Emmanuel Thibert (ingénieur de recherche à l'IRSTEA), Cédric Dentant (chargé de mission flore-habitat au service scientifique du Parc national des Écrins, pilote du programe CCLimaT). À Joseph Langridge (chargé de mission revue systématique au sein de l'UMS PatriNat). À nos amis malacologues pour leur participation active tant sur le cadre conceptuel d'étude des Mollusques sur ORCHAMP que sur la mise en ouvre des études sur le terrain, Alain Thomas, Christophe Perrier et Nicolas Bertrand. Nous remercions également Xavier Cucherat et Lilian Léonard qui ont enrichi ce travail par leur relecture. 


\section{RÉFÉRENCES}

Baurand P.-E., Capelli N., Scheifler R. \& Vaufleury A. 2014. - An assessment of the embryotoxicity of cadmium in the terrestrial mollusk Cantareus aspersus: from bioaccumulation to impacts at different levels of biological organization. Ecotoxicology and Environmental Safety 110: 89-94. https://doi.org/10.1016/j. ecoenv.2014.08.017

Combrisson D. \& Maillard D. 2016. - État des lieux sur la connaissance de la malacofaune du Parc national des Écrins: l'exemple de l'Embrunais. MalaCo 12: 12-16.

GREC-SUD 2018. - Impacts du changement climatique et transition(s) dans les Alpes du Sud. Les cahiers du GREC-SUD, Association pour l'innovation et la recherche au service du climat (AIR), Marseille, $48 \mathrm{p}$.

Hall C., Rhind S. \& Wilson M. 2009. - The potential for use of gastropod molluscs as bioindicators of endocrine disrupting compounds in the terrestrial environment. Journal of Environmental Monitoring: JEM 11: 491-7. https://doi.org/10.1039/b804320e

LIMONDIN-LOZOUET N. 2011. - Successions malacologiques à la charnière Glaciaire/Interglaciaire: du modèle TardiglaciaireHolocène aux transitions du Pleistocène. Quaternaire 22: 211220. https://doi.org/10.4000/quaternaire.5971

Louzon M., Zahn S., CaPelli N., Massemin S., Coeurdassier M., Pauget B., Gimbert F. \& Vaufleury A. 2020. — Impact of ageing and soil contaminants on telomere length in the land snail. Ecotoxicology and Environmental Safety 201: 110766. https://doi. org/10.1016/j.ecoenv.2020.110766

MaGNIN F. \& MARTIN S. 2008. — Grandeur et misère de l'analyse malacologique, ou comment discriminer les facteurs climatiques et anthropiques de l'évolution des paysages holocènes. Bulletin archéologique de Provence supplément 5: 61-73.

Magnin F., Tatoni T., Roche P. \& Baudry J. 1995. - Gastropod communities, vegetation dynamics and landscape changes along an old-field succession in Provence, France. Landscape and
Urban Planning 31: 249-257.

Martin S., Jorda C., Gandelin M., Jung C. \& Bel V. 2017. L'étude des escargots au service de l'archéologie ou l'apport de la malacologie à la compréhension des activités agro-pastorales et des paysages anciens. Les nouvelles de l'archéologie 148: 34-39. Müller J., STRÄTz C. \& HothorN T. 2005. - Habitat factors for land snails in European beech forests with a special focus on coarse woody debris. European Journal of Forest Research 124: 233-242. https://doi.org/10.1007/s10342-005-0071-9

PeARCE T. \& PAUSTIAN M. 2013. - Are temperate land snails susceptible to climate change through reduced altitudinal ranges? A Pennsylvania example. American Malacological Bulletin 31 (2): 213-224. https://doi.org/10.4003/006.031.0201

Puisségur J., Limondin-LOZOUET N. \& Rousseau D.-D. 1994. Changements environnementaux et climatiques en Bourgogne à l'Holocène à partir de l'étude des mollusques continentaux [Environmental and climatic changes derived from Holocene malacofaunas from Burgundy, France]. Quaternaire 5: 189-190. https://doi.org/10.3406/quate.1994.2031

Rousseau D., Limondin-Lozouet N. \& Puissegur J. 1992. Réponses des assemblages malacologiques holocènes aux impacts climatiques et anthropiques sur l'environnement. Comptes rendus de l'Académie des Sciences 315: 1811-1818.

Thompson I., Mackey B., McNulty S. \& Mosseler A. 2009. Forest resilience, biodiversity, and climate change. A synthesis of the biodiversity/resilience/stability relationship in forest ecosystems. Secrétariat de la Convention sur la diversité biologique, Montréal. Cahier technique 43: 1-67.

VAUfLEURY A. 2015. — Landsnail for ecotoxicological assessment of chemicals and soil contamination - ecotoxicological assessment of chemicals and contaminated soils using the terrestrial snail, Helix aspersa, at various stage of its life cycle: a review. Environmental Indicators 365-391. https://doi.org/10.1007/97894-017-9499-2_23 